\title{
REAL TIME TRAJECTORY CORRECTION SYSTEM OF OPTICAL HEAD IN LASER WELDING
}

\author{
Wojciech CIESZYŃSKI", Michał ZIĘBA*, Jacek REINER* \\ *Department of Laser Technology, Automation and Organization of Production, Wroclaw University of Technology, \\ Wybrzeże Wyspiańskiego 27 50-370 Wrocław, Poland \\ wojciech.cieszynski@pwr.edu.pl, zieba.nowice@gmail.com, jacek.reiner@pwr.edu.pl \\ received 1 October 2014, revised 17 December 2015, accepted 18 December 2015
}

\begin{abstract}
Application of laser welding technology requires that the laser beam is guided through the whole length of the joint with sufficiently high accuracy. This paper describes result of research on development of optomechatronic system that allows for the precise positioning of the laser head's TCP point on the edge of welded elements during laser processing. The developed system allows for compensation of workpiece's fixture inaccuracies, precast distortions and workpiece deformations occurring during the process.
\end{abstract}

Key words: Control System, Machine Vision, Image Processing, Laser Welding

\section{INTRODUCTION}

High precision motion in the process of laser welding involves integration of the positioning systems that control the trajectory of the laser spot with respect to the processed weld, and are able to adjust the position of the laser head. Positioning systems improve flexibility of the processing site, admit greater diversion from the assumed dimensions of the welded pieces, save time and improve quality of the assembly.

The research was aimed at development of a video positioning system for the laser head operating with the CNC machine that would improve accuracy of the trajectory by mean of real time compensation of the assembly errors of the welded pieces in the machine system.

\section{CURRENT KNOWLEDGE}

There were a number ideas devised over last decades of how to position the laser head. These include: (a) scanning with a pointed laser beam (Fridenfalk and Bolmsjö, 2003), b) optical recognition of the joint on a camera image taken in advance, c) triangulation with sensors measuring the distortions of the projected light profiles, d) mechanical tracking of the seam (Siciliano and Khatib, 2008).

The industry most wide spread solutions involve triangulation. These systems control the seam with $0.1 \mathrm{~mm}$ accuracy for straight line trajectories and $0.5 \mathrm{~mm}$ for the curved ones. The limited resolution arises from the inaccuracy of the image processing and the sensors' resolution (De Graaf et al., 2005, 2007, 2010). Triangulating scanners are used also for the postprocessing quality inspection (Huang and Kovacevic, 2011, 2012).

There are also extended triangulation methods - stereo triangulation (Michalos et al., 2012) where trajectory are adjusted in 3D for manifold surfaces. Michalos et al. (2012) show how a camera system is integrated on a robotic arm designed for welding. The system has been implemented in the automobile doors assembly line.

Some machine vision positioning systems determine position using the information content of images recorded with an industrial camera mounted on the laser head. A system analysing infrared $(805-815 \mathrm{~nm})$ images of a molten pool, provides for a $60 \mu \mathrm{m}$ accuracy. This, however, works with the pulse lasers only, as the camera records images between the pulses.

Gao et al. (2013) proposed a method analysing the shape of the pool's front with the camera using a narrow $960-990 \mathrm{~nm}$ spectrum. The joint edge is being detected on the images taken during the welding, and the data used to adjust the trajectory of the laser head.

There is a trend to integrate the positioning system with molten pool monitoring and quality control of the seam (Dorsch et al., 2013). These methods use coaxial triangulation in front of and in the rear of the molten pool and the keyhole imaging. Thanks to the application of a infrared spectrum filter and the monochromatic projectors, the images can show both the triangulation profiles and the pool itself. These solutions are applied in the systems of laser welding of pipes and sectional metal bars.

\section{DESIGN OF THE POSITION ADJUSTING SYSTEM FOR THE BEAM/LASER HEAD}

\subsection{Concept}

The processing under consideration makes a ring shaped laser welded seam. This requires the laser head travelling along a full ring trajectory. The proposed system makes use of a stationary laser head and a rotating table of the machine.

The proposed adjustment system follows the diversions of the trajectory from the assumed shape and position. Those are detected by the optical sensor when they enter the scanning field moving in advance of the true laser beam at the preset distance. Diversions of the true joint edge from the assumed position are computed from the position of the joint edge detected in the sen- 
sor's scanning field. The results are used to control the motion of the machine working head (Fig. 1).

a)

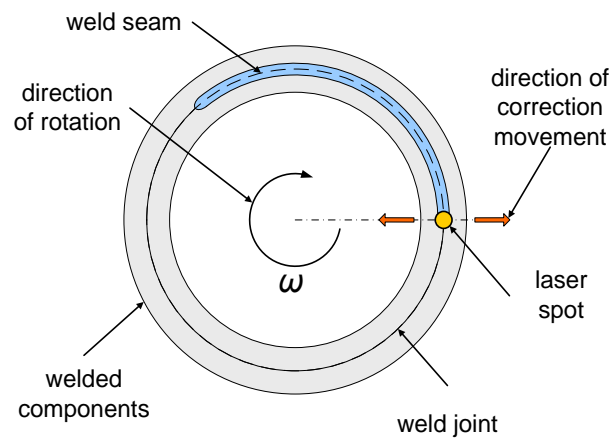

b)

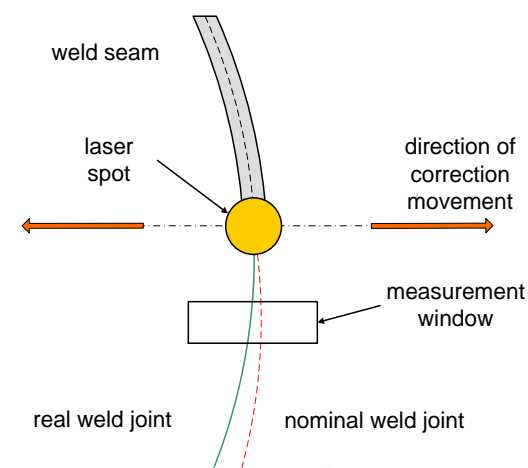

Fig. 1. Laser beam positioning system: a) general concept; b) detailed view

The adjustment motions of the machine head are perpendicular to the ideal, reference trajectory of the laser head in a coordinate system attached to the machine's table. This way, the adjustment movements do not combine with the linear speed of the head controlled in the processing script.

Computation of the adjustment signal rely on the adopted kinematic model that reflects the assumed trajectory and the motions that are drawing it.

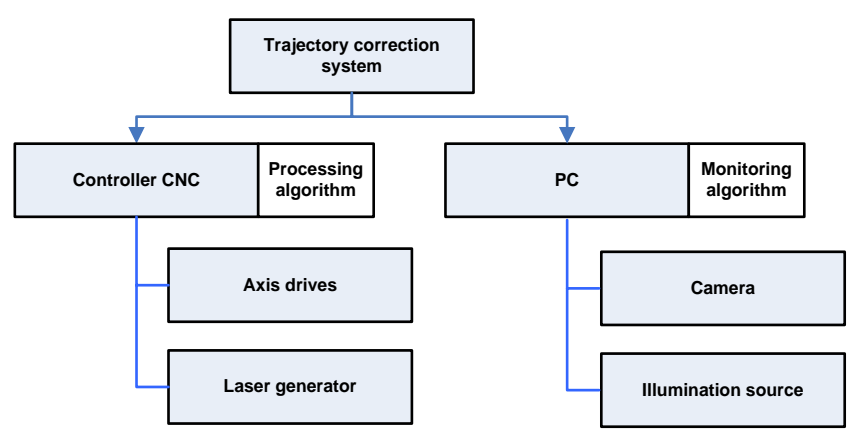

Fig. 2. Structure of the trajectory adjustment system

The concept assumes that the control algorithm will run along two parallel paths: the CNC processing script controlling the manufacturing process, and the adjustments algorithm which supervises the process and computes the adjustments, running on a PC communicated with the CNC controller (Fig. 2).
The algorithm executed on the machine includes the main technology task of controlling the kinematic system which, in turn, draws the assumed trajectory, as well as controlling of the laser beam during the machine processing. Except for that, the programme includes the elements activating and effecting communication between the machine and the PC, and the commands triggering the adjustment motions superimposed on the trajectory.

The other algorithm running on the PC supervises the process and generates the adjustment signals. Thus it processes the images created by the camera and retrieves from the machine the data concerning the head position and the job progress. Using the collected information the program computes the diversion of the machine head from the assumed position and signals the adjustments to the CNC controller in the machine.

\subsection{Stages of operation}

There are three specific images of the operating field that are distinguishable when a closed curve shaped seam is being drawn: (1) when only the gap is visible, (2) when the gap and the molten pool are visible, (3) when the molten pool and front of the seam are visible - the closure of the trajectory. Since there are varying algorithms of image analysis in use, 3 stages of operation have been distinguished (Fig.3).

a)

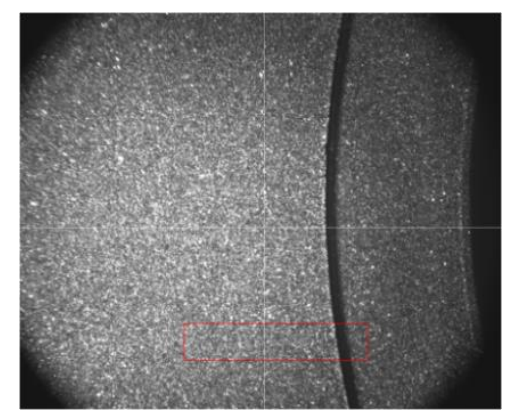

b)

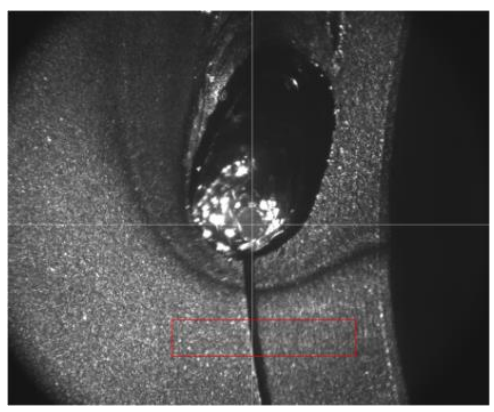

c)

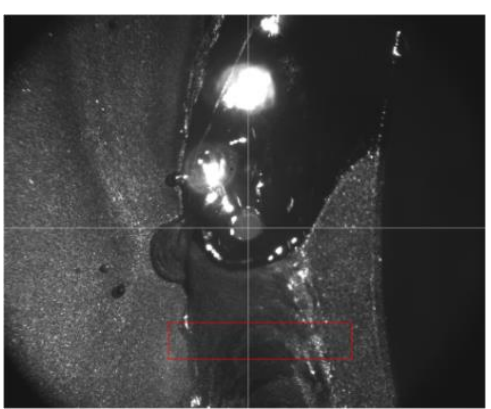

Fig. 3. Images of the elements at the subsequent process stages: a) initial stage; b) processing stage; c) finishing stage 
Initial stage (Fig. 3a) - includes the table rotation when the images of the gap are recorded and the head motion adjustments determined. At this stage, the laser beam is not in use and no melting is affected. The resulting control signals are fed to the machine input thus controlling the machine head position. At the same time, the generated signals are recorded in the buffer for the later use, when the trajectory is being closed. At this stage, the table turns by 10 - 20 degrees.

Processing stage (Fig. 3b) - begins after the table reaches the assumed angle determined by the angular fore of the scanning field with respect to the laser beam angular position. The control signals that are now being computed basing on the recorded images and the current position of the beam, are fed to the CNC controller. At the start of with this stage, the laser induced melting begins. The end of this stage is marked with the table travelling the full circle from the initial position in the stage 1.

Finishing stage (Fig. $3 \mathrm{c}$ ) - now the control signals are not generated upon the current images, but fed from the buffer where they were stored in the initial stage. The third stage ends when the full length of the seam is ready.

\subsection{Hardware configuration}

The operation site consisted of the following components: CNC machine, PC, data acquisition adapter, AC/DC, industrial CMOS camera, illumination (810nm laser).

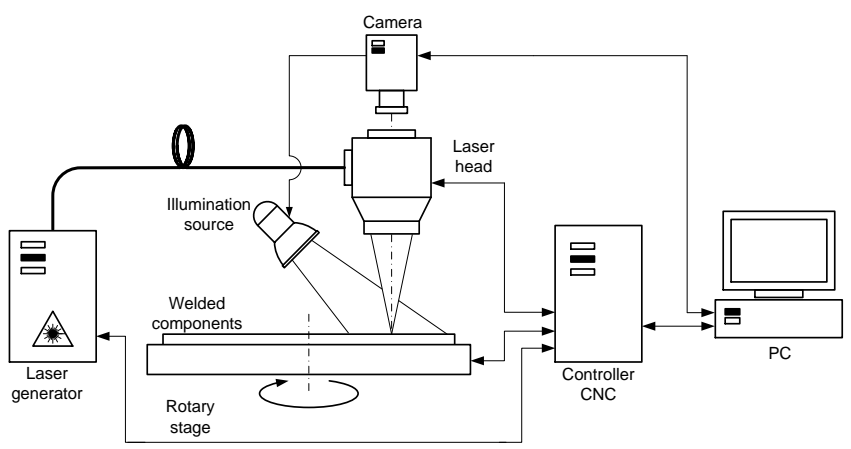

Fig. 4. Head positioning system - diagram

The following signals had to be communicated between the CNC controller and the supervising PC: reading of the laser head position data, reading by the CNC controller of the positioning diversion, reading by the $\mathrm{PC}$ of the synchro signals. The data concerning the position and the diversion are transferred by the analogue communication modules available in the machine control system (Siemens, 2006). The synchro signalling went through the digital output of the PLC controller that was a part of the CNC controller.

\section{DETECTION OF THE JOINT EDGE}

The edge detection algorithm processes images and then fits the curve to the edges detected on the image.

The first stage of image processing defines the region of interest (ROI) on the recorded image. A window set in the right position facilitates more effective image processing, limited to a piece of the the recorded image, where the junction edge and the molten pool are visible. The position of the window has been so adjusted, as to avoid the disturbances caused by the steam channel, the molten pool and the area of heat escape.

a)

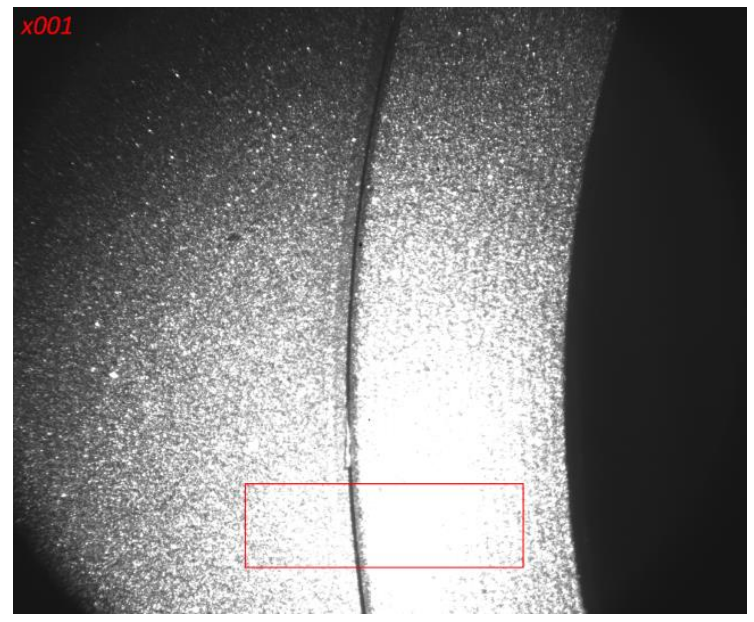

b)

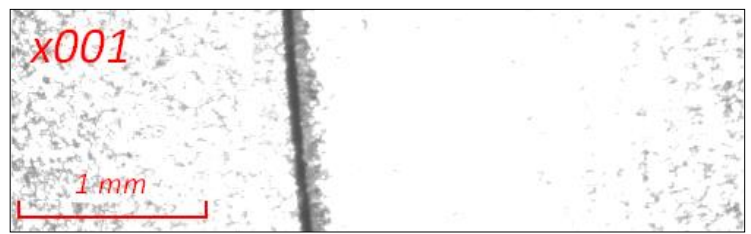

c)

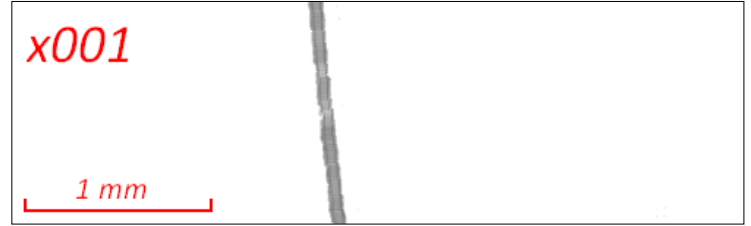

d)

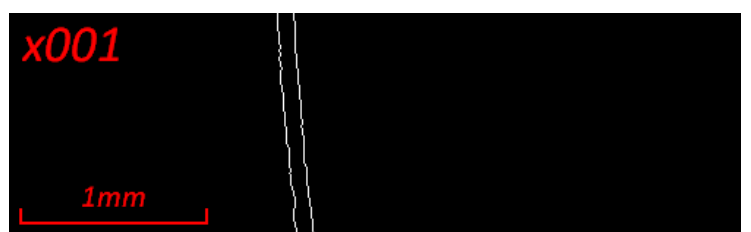

Fig. 5. Image processing: a) base image with ROI window; b) processed piece of the image; $c$ ) the image after filtering; d) the image after processing

a)
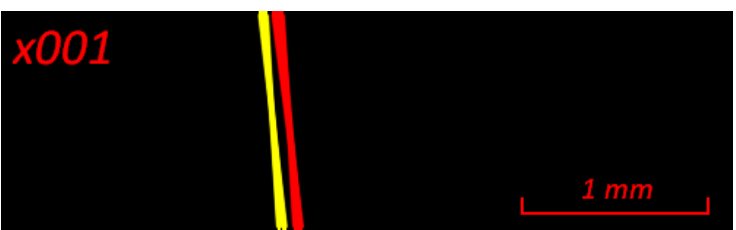

b)

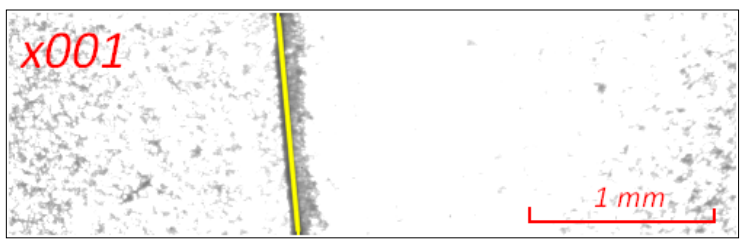

Fig. 6. Results of the edge detection algorithm with straight line model: a) edges detected with the Hough transform; b) net outcome straight line of the seam

The joint edge is visible as brighter line among the dark surroundings. In the beginning the images are subjected to filtering 
(median, and Gauss), morphology (erosion, opening and closing) and histogram operations (adjustment of brightness, contrast) in order to remove the distortions arising from the brightness of the surface processed. Fig. 5c).

Next, the resulting image is gradient filtered to provide the ROI edges, used further by the algorithm (Fig. $5 \mathrm{~d}$ ), with visible edges of the pieces to be welded. The images showing the detected edges are used for straight line approximation of joint edge. Hough transform [14] provides for the set of lines fit to the edges visible on the image (Fig. 6a). The net fit to the joint edge is computed by averaging (Fig. 6b).

\section{DETERMINATION OF THE HEAD POSITION ADJUSTMENTS}

The adjustment signal is computed in a model accounting for the machine kinematics, shape of the processed item, and its position in the machine operating space.

The coordinate system is stationary with respect to the machine's body and located at the centre of the table rotations $P_{0}$, while the angular velocity of the table is constant. Coordinates of the head and the lines tracing the joint on the recorded images are relative to the $P_{0}$. The adjustment motion vectors are parallel to the $X$ axis of the coordinate system, and the camera see this axis as a horizontal line.

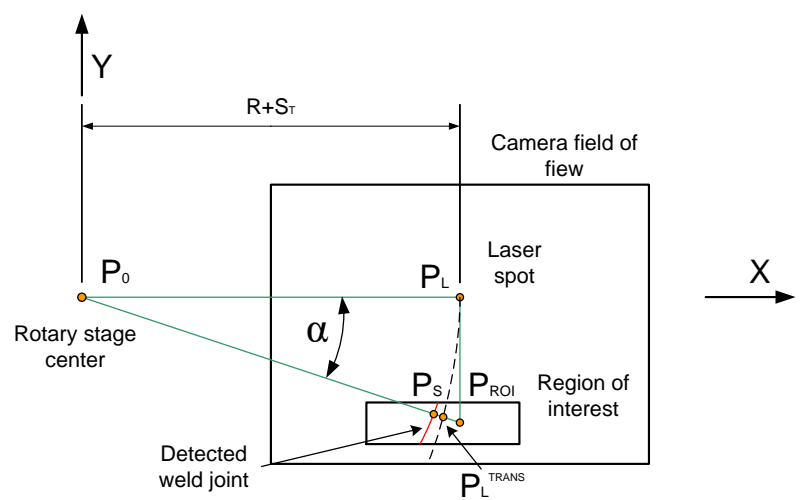

Fig. 7. The coordinate systems used in computations

A point $P_{L}$ representing location of the laser beam has two coordinates assigned on the image. A $P_{R 0 I}$ point is determined in the $R O I$ window. Its $X$ coordinate is equal to that of $P_{L}$ while the $Y$ coordinate equals a half of the $R O I$ window height. The points $P_{0}, P_{L}, P_{R 0 I}$ make a right triangle used for computations (Fig. 7).

Position of $P_{L}$ and $P_{R 0 I}$ in the image is determined manually in advance of the process and then, the adjustment signals change the $X$ component of the distance between the $P_{L}$ and $P_{0}$ as compared with the nominal value $R$.

$\left|\overline{P_{0} P_{L}}\right|=R+S_{T}$

where: $x$ - description, $a$ - description, $a^{n-k}-$ description, $\left(\begin{array}{l}n \\ k\end{array}\right)$-description.

The points $P_{0}$ and $P_{R 0 I}$ are positioned on the straight line along which the adjustment of the head position are computed. For each measurement cycle, the line representing the joint with the $P_{0}, P_{R 0 I}$ line determine the point $P_{S}$ of the assumed next position of the seam (Fig. 7).
The lasers' point of work $\mathrm{P}_{\mathrm{L}}$, is rotated around the $P_{0}$, and this way projected on the $\left|\overline{P_{0} P_{L}}\right|$, axis, what provides for the next position of the point of laser $P_{L}^{T R A N S}$ relating to the current signal $S_{T}$. $P_{L}^{T R A N S}$ distance from $P_{R O I}$ for a given angle of forwarding is determined as:

$\left|\overline{P_{L}^{T R A N S} P_{R O I}}\right|=\left(R+S_{T}\right) \cdot\left(\frac{1}{\cos (\alpha)}-1\right)$

where: $x$ - description, $a$ - description, $a^{n-k}$ - description, $\left(\begin{array}{l}n \\ k\end{array}\right)$-description.

The difference between the $P_{S}$ and $P_{L}^{\text {TRANS }}$ distances to the

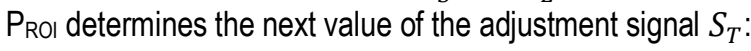

$S_{\Delta T}= \pm\left(\left|\overline{P_{L}^{T R A N S} P_{R O I}}\right|-\left|\overline{P_{S} P_{R O I}}\right|\right)$

An adjustment signal thus computed cannot be as yet sent to the CNC Controller. The adjustment signal shall provide the target position of the machine head at the given time, instead of the translation increments relating to the computed value of the adjustment signal $S_{\Delta T}$. Therefore, the base signal representing the position of the head at the time of measurement shall be added $S_{T}$

$S_{T+\Delta T}=S_{T}+S_{\Delta T}[u m]$

The time of measurement $\Delta T$ pre-emption is computed from the values of the $\alpha$ angle and the table angular velocity $\omega$ assumed for the welding process.

$\Delta T=\frac{\alpha}{\omega}[s]$

\section{SYSTEM OPERATION CHECK}

The system operation has been assessed comparing the head trajectory with and without use of the positioning system. The processed item with a random imposed diversion from the base position was set up on the machine table.

Two job cycles with active laser beam were run:

- the first was an initial run with no positioning system, when the head travelled along the base trajectory, for the purpose of recording of the images used for computation of the head diversion from the joint edge;

- the second was a test run with an active positioning system. During the second run, the photo shots were taken which facilitate assessment of the accuracy of the laser beam with respect to the joint edge.

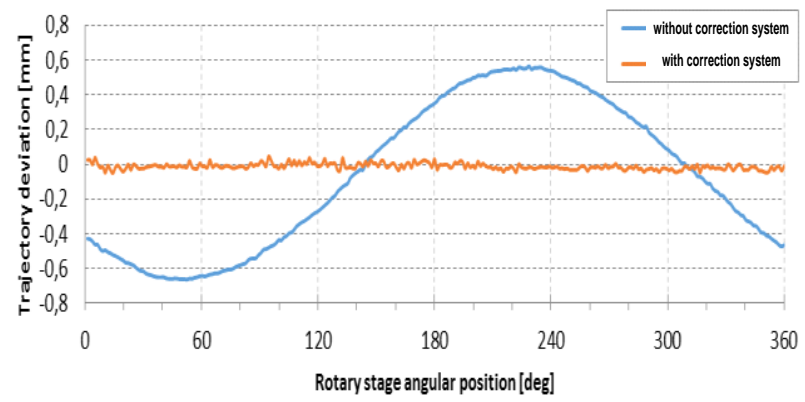

Fig. 8. Comparison of the head diversion from the position of the joint with active and inactive adjustment system (processing parameters: laser power $2 \mathrm{~kW}$, table rotation speed $45 \%$ ) 
The Fig. 8 chart shows the diversion of the head position with respect to the point of junction, recorded during the initial run without the adjustment system and then with active adjustment system. The diversion of the head position is computed as the distance between the junction point in the gap between the edges and the point of operation of the laser.

The test item showed diversions of $0.66 \mathrm{~mm}$ between the gap and the laser head position. The data gathered during the assessment run show that the adjustment system reduced the positioning error to $50 \mu \mathrm{m}$.

\section{SUMMARY}

The designed system of the real time laser head position adjustment provides for the more accurate welding process and elimination of the impact of the inaccurate assembly of the processed item on the seam accuracy.

The positioning accuracy of $50 \mu \mathrm{m}$ achieved in the system assessment runs is several times better than the diameter of laser beam dots used for welding (about $600 \mu \mathrm{m}$ ). This provides for the proper adjustment of the laser position at about $10 \%$ with respect to the dot diameter. With this configuration, the accuracy of positioning of the kinematic system of the CNC machine becomes a leading factor in the overall accuracy.

Overall operation of the process monitoring system depend on types and shapes of the joints, the process parameters, the surface texture of the welded pieces, illumination, technology requirements and many other factors. This is why these systems are dedicated and further development may increase flexibility of their application.

The designed system strong points include detection of the joint traces where the gap between the pieces is narrower than accuracy of the triangulating sensors. Laser illumination allows for the process monitoring what is troublesome with traditional approach because of strong disturbances from plasma and process gases. This way the impact of the parametrisation on the process performance can be assessed, especially at the ends, where the seams are most prone to defects.

\section{REFERENCES}

1. De Graaf M. (2007), Sensor-guided robotic laser welding, University of Twente.

2. De Graaf M., Aarts R., Meijer J., Jonker J.B. (2005), Robot-sensor synchronization for real-time seam tracking in robotic laser welding, Proceeding of the Third International WLT-Conference on Lasers in Manufacturing, Munich.

3. De Graaf M., Aarts R., Jonker J.B., Meijer J. (2010), Real-time seam tracking for robotic laser welding using trajectory-based control, Control Eng. Pract., Vol. 18, No. 8, 944-953.

4. Dorsch F., Pfitzner D., Braun H. (2013), Improved continous tube welding due to unique process sensor system and process control, Phys. Procedia, Vol. 41, 137-139.

5. Fridenfalk M., Bolmsjö G. (2003), Design and validation of a universal $6 \mathrm{D}$ seam tracking system in robotic welding based on laser scanning, Industrial Robot: An International Journal, Vol. 30 No. 5, 437-448.

6. Gao X., Zhong X., You D. (2013), Kalman Filtering Compensated by Radial Basis Function Neural Network for Seam Tracking of Laser Welding, IEEE Trans. Control Syst. Technol., Vol. 21, No. 5, 19161923

7. Huang W., Kovacevic R. (2011), A Laser-Based Vision System for Weld Quality Inspection, Sensors, Vol. 11, 506-521.

8. Huang W., Kovacevic R. (2012), Development of a real-time laserbased machine vision system to monitor and control welding processes, The International Journal of Advanced Manufacturing Technology, Vol. 63(1), 235-248.

9. Lee S.K., Na S. J. (2012), A study on automatic seam tracking in pulsed laser edge welding by using a vision sensor without an auxiliary source, Journal of Manufacturing Systems, Vol. 21(4), 302-315

10. Michalos G., Makris S., Eytan A., Matthaiakis S., Chryssolouris G. (2012), Robot Path Correction Using Stereo Vision System, Procedia CIRP, Vol. 3, 352-357.

11. Rafajłowicz E., Rafajłowicz W., Rusiecki A. (2009), Image processing algorithms and an introduction to working with the OpenCV library (in Polish), Wroclaw University of Technology Press.

12. Regaard B., Kaierle S., Poprawe R. (2009), Seam-tracking for high precision laser welding applications - Methods, restrictions and enhanced concepts, Journal of Laser Applications, Vol. 21 (4), 841875.

13. Siciliano B., Khatib O. (eds.) (2008), The Handbook of Robotics, Springer, Berlin, Heidelberg.

14. Siemens AG. (2006), SINUMERIK 840D Configuring the NCU.

The project has been subsided by the European Union within the European Regional Development Fund Priority axis 1. Strengthening research, technological development and innovation; "Design of a new generation automobile seat in Sitech company (WND-POIG.01.04.00-02067/12).

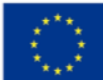

